\title{
Avaliação de Impacto à Saúde (AIS) no Brasil e América Latina: uma ferramenta essencial a projetos, planos e políticas
}

Karina Camasmie Abe $\mathrm{Ab}^{(\mathrm{a})}$

Simone Georges El Khouri Miraglia(b)

Abe KC, Miraglia SGEK. Health Impact Assessment (HIA) in Brazil and Latin America: an essential tool for projects, plans and policies. Interface (Botucatu). 2018; 22(65):349-58.

The paper presents a literature review of the application of the Health Impact Assessment (HIA) methodology, disseminated by the World Health Organization in Brazil and Latin America. This study showed that the practice and application of the HIA is not common in Brazil or Latin America, as well as the analysis of health impacts is carried out in a superficial way regarding environmental licensing and public policies, indicating the need of the use of specific health impact assessment methodologies, professional training and government initiative. The adoption of HIA in Brazil could serve to avoid adverse health effects and could enhance the positive aspects, as well as to mitigate the negative aspects of projects and public policies, ensuring that people's health is not neglected.

Keywords: Health impact assessment. Public health. Brazilian National Health System. Health management. Brazil.
O presente artigo apresenta uma revisão de literatura e discute a aplicação da metodologia de Avaliação de Impacto à Saúde (AIS), divulgada pela Organização Mundial de Saúde (OMS), para o Brasil e América Latina. Esse estudo mostrou que a prática e a aplicação da AIS não é comum nestas regiões, e, além disso, a análise de impactos à saúde é realizada de forma superficial em licenciamentos ambientais e em políticas públicas, o que aponta para a necessidade da utilização de metodologias específicas, treinamento de profissionais e iniciativa governamental. A adoção da AIS no Brasil e América Latina evitaria a geração de efeitos adversos à saúde, potencializando os aspectos positivos, mitigando os efeitos negativos de projetos e políticas públicas, garantindo que a saúde da população não seja negligenciada.

Palavras-chave: Avaliação do impacto na saúde. Saúde pública. Sistema Único de Saúde. Gestão em saúde. Brasil.

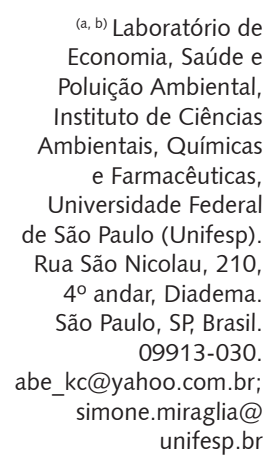

(a, b) Laboratório de Economia, Saúde e Poluição Ambiental, Instituto de Ciências Ambientais, Químicas e Farmacêuticas, Universidade Federal de São Paulo (Unifesp). Rua São Nicolau, 210, $4^{\circ}$ andar, Diadema. São Paulo, SP, Brasil. 09913-030. abe_kc@yahoo.com.br; simone.miraglia@ unifesp.br 


\section{Introdução}

A Avaliação de Impacto à Saúde ou Avaliação do Impacto na Saúde (AIS) tem como marco inicial o documento chamado 'Consenso de Gotemburgo', publicado em 1999, pela Organização Mundial de Saúde (OMS)'. Tal instrumento descreve os conceitos principais e as abordagens possíveis, no âmbito local, nacional ou internacional. Em suma, a AIS é uma ferramenta que combina métodos quantitativos e qualitativos, atendendo a uma abordagem multidisciplinar, que considera uma vasta gama de efeitos à saúde ${ }^{1-4}$. Anteriormente à publicação do Consenso de Gotemburgo, diversos estudos sobre avaliações de impactos à saúde foram realizados, no entanto, as metodologias aplicadas eram muito diversificadas, o que reforçou a necessidade da padronização de técnicas, etapas e análises de efeitos à saúde, possíveis de serem replicadas e aplicadas internacionalmente.

Para se entender a origem da AIS, também é necessário compreender o papel e a história do Estudo de Impacto Ambiental (EIA). O EIA surgiu há quase quatro décadas, nos EUA, a partir do The National Environmental Policy Act (NEPA), uma lei promulgada em 1969 e que vigorou a partir de 19705,6. Os idealizadores do EIA viam nele um instrumento político para forçar uma mudança de comportamento, de modo a atender demandas da sociedade para compatibilizar atividades econômicas e defesa ambiental. Assim, essa ferramenta proporcionou grandes avanços na área ambiental, mas também foi constante alvo de críticas? ${ }^{7}$.

No Brasil, a origem do EIA seguiu a tendência verificada para países em desenvolvimento e está relacionada à necessidade de infraestrutura e logística na implementação de projetos de grande porte, a exemplo de polos industriais, petroquímicos, usinas hidrelétricas e demais empreendimentos

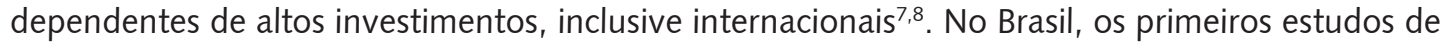
avaliação de impacto ambiental foram os das barragens de Sobradinho, no Rio São Francisco (1972), e Tucuruí, no Rio Tocantins (1977), ambos financiados pelo Banco Mundial7.

O EIA-RIMA (Estudo de Impacto Ambiental e seu respectivo Relatório de Impacto Ambiental) foi regulamentado nacionalmente, a partir da Resolução CONAMA 001, de 23 de janeiro de $1986^{9}$, e foi um dos instrumentos da Política Nacional do Meio Ambiente (PNMA $)^{10}$ para garantir a realização da Avaliação de Impacto Ambiental (AIA), cujo objetivo era a defesa do meio ambiente em projetos de grande escala. Essa resolução, em seu Artigo $1^{\circ}$, também estabeleceu a definição de impacto ambiental: "considera-se impacto ambiental qualquer alteração das propriedades físicas, químicas e biológicas do meio ambiente, causada por qualquer forma de matéria ou energia resultante das atividades humanas que, direta ou indiretamente, afetam: I - a saúde, a segurança e o bem-estar da população; II - as atividades sociais e econômicas; III - a biota; IV - as condições estéticas e sanitárias do meio ambiente; $\mathrm{V}$ - a qualidade dos recursos ambientais" (grifo nosso). Adicionalmente, em seu Artigo 6º a Resolução Conama $n^{\circ}$ 09, de 3 de dezembro de 1987, estabeleceu os critérios mínimos de desenvolvimento das atividades técnicas do EIA-RIMA, o que inclui o diagnóstico ambiental de influência do projeto, abrangendo: o meio físico; o biológico e os ecossistemas naturais, além do meio socioeconômico ${ }^{11}$.

A consideração desses elementos pelos EIA-RIMAs é um aspecto importante, visto que a saúde populacional possui vários determinantes sociais e ambientais, inclusive considerados na Resolução CONAMA no 01/86 ${ }^{\circ}$. Além disso, de acordo com o Artigo 19 da Resolução CONAMA n 237/97, é permitido, ao órgão ambiental, suspender ou cancelar uma licença, em caso de superveniência de graves riscos ambientais e de saúde ${ }^{12}$. No entanto, na prática, os estudos exigidos no licenciamento ambiental se preocupam, majoritariamente, com os impactos diretos ao meio ambiente e à saúde, não são explicitados ${ }^{13}$. Essa questão é uma das principais críticas que o EIA-RIMA sofreu posteriormente à sua implantação e, no Brasil, a negligência em relação aos efeitos à saúde provoca a sobrecarga no Sistema Único de Saúde (SUS) e aumento dos custos associados, favorecendo as inequidades na saúde populacional. Surgiu, assim, a necessidade de se ampliar e aprofundar a análise de impactos ambientais e em saúde, inserindo estudos e metodologias focados neste setor. Uma das metodologias aplicadas em países em desenvolvimento e que será discutida neste artigo é a AIS (sigla em português e HIA Health Impact Assessment, em inglês).

Portanto, essa revisão de literatura objetiva verificar as publicações brasileiras e latinas sobre a aplicação da AIS ${ }^{1}$, e se propõe, ainda, a discutir o tema em consonância aos princípios do SUS, com 
o intuito de subsidiar a aplicação desse conjunto de metodologias em projetos, planos e políticas no Brasil. A análise desenvolvida não tem a pretensão de ser exaustiva. Trata-se, antes, de um exercício de síntese e de discussão de perspectivas sobre o tema.

\section{Metodologia}

A metodologia desse estudo consistiu em uma revisão da literatura, baseada na utilização da AIS no Brasil e na América Latina. As publicações foram selecionadas por meio de buscas nas bases de dados: Medline (PubMed), Literatura Latino-Americana e do Caribe, em Ciências da Saúde (Lilacs), Scientific Eletronic Library Online (SciELO) e Biblioteca Virtual em Saúde (BVS), entre agosto de 2015 e fevereiro de 2016. O período de publicação considerado foi entre os anos de 1999 e fevereiro de 2016, em língua inglesa, portuguesa ou espanhola, e que tratassem da aplicação da metodologia da AIS, como preconizado pela OMS, no Brasil ou América Latina. Os descritores em saúde associados nas buscas das publicações foram os seguintes: em inglês: 'Health Impact Assessment'; Brazil; 'Latin America' e 'South America'; em português: 'Avaliação do Impacto na Saúde'; 'Avaliação de Impacto à Saúde', Brasil; e em espanhol: 'Evaluación del Impacto em la Salud'. Foram também consideradas teses ou dissertações, assim como diretrizes internacionais e nacionais relevantes sobre o tema. Além das publicações, foi realizada uma ampliação a respeito da metodologia da AIS e seus princípios, relacionando-a aos princípios do SUS, embasando-se na legislação brasileira e nos capítulos de livros sobre o tema. Três categorias temáticas, discutidas a seguir, foram apreendidas a partir da análise das publicações.

\section{AIS e Licenciamento Ambiental no Brasil}

A AIS não é institucionalizada em nenhum país da América Latina ${ }^{14}$ e, apesar de ainda não ser aplicada em projetos ou políticas públicas no Brasil, o respaldo para a sua adoção em projetos possui base na própria regulamentação e legislação brasileiras. A AIA, que depende da elaboração do EIARIMA, foi a primeira ferramenta de política ambiental de uso corrente no Brasil, implantada como um dos instrumentos da PNMA, pela Lei $n^{\circ}$. 6.938/81 $11^{10}$. Mas foi a partir da Resolução Conama $n^{\circ}$. 01, de 23 de janeiro de $1986^{9}$, que se vinculou a AIA e, respectivamente, o EIA-RIMA ao licenciamento ambiental de atividades potencialmente poluidoras.

A institucionalização e obrigatoriedade destes estudos significou um marco na evolução do ambientalismo brasileiro, uma vez que, anteriormente a essas resoluções, os grandes empreendimentos apenas consideravam variáveis técnicas e econômicas, sem a inclusão das ambientais e sociais ${ }^{15}$. Outro fator relevante é que o licenciamento ambiental possui a característica de incluir a participação popular na tomada de decisão, por meio de audiências públicas ${ }^{9}$, ponto também importante no processo da AIS ${ }^{14,16,17}$.

Apesar de a Resolução Conama no 001/86 considerar impacto ambiental qualquer alteração das propriedades físicas, químicas e biológicas do meio ambiente, resultante das atividades humanas que, direta ou indiretamente, afetem a saúde, a segurança e o bem-estar da população, assim como as atividades sociais e econômicas ${ }^{9}$, a participação do setor saúde no licenciamento ambiental é incipiente ${ }^{18}$. É importante mencionar que não existe regulamentação de nenhuma ferramenta específica de avaliação de impactos à saúde, para o processo de licenciamento ambiental ${ }^{15}$.

Adicionalmente, o estudo de Silveira et al., verificou que, de 36 estudos ambientais direcionados ao setor saúde pelo órgão ambiental federal competente (Instituto Brasileiro do Meio Ambiente e dos Recursos Naturais Renováveis - IBAMA), apenas 50\% tiveram alguma recomendação incorporada como condicionante às licenças ambientais ${ }^{15}$. Muitas das questões incorporadas do setor saúde limitam-se, única e exclusivamente, à questão de acidentes dos trabalhadores e à infecção por malária, quando o empreendimento se localiza em áreas endêmicas dessa doença ${ }^{15,19}$. Essa abordagem está longe de contemplar a saúde de forma sistemática e como um estado de completo bem-estar, físico, mental e social, e não meramente ausência de doenças ${ }^{20}$. Considerando isso, os órgãos ambientais responsáveis pelo processo de licenciamento ambiental não estão preparados para analisarem a 
saúde nessa conjectura, também existindo limitações de natureza técnica e de infraestrutura, além de dificuldades institucionais de gestão para o estabelecimento de um processo integrado de vigilância e controle dos aspectos de ambiente e saúde ${ }^{13}$.

A negligência com os efeitos à saúde da população ocorre com relativa frequência, uma vez que os únicos mecanismos legalizados de participação da saúde no processo de licenciamento ambiental se dão por meio da Resolução CONAMA 286/2001, que regulamenta o licenciamento de empreendimentos em regiões endêmicas de malária ${ }^{21}$, e da Portaria $n^{\circ} 47$ de 29 de dezembro de $2006^{22}$, que dispõe sobre a Avaliação do Potencial Malarígeno e o Atestado de Condição Sanitária para projetos de assentamento de reforma agrária e outros empreendimentos em regiões amazônicas ${ }^{13,18}$. Para outros determinantes de saúde, não há legislação ou diretrizes específicas atreladas ao licenciamento ambiental.

Vale ressaltar que a AIS pode ser útil quando aplicada em grandes empreendimentos, mas não se limita unicamente a essa grande área. A AIS pode também ser aplicada em projetos de pequeno porte e para análises de políticas públicas ${ }^{23}$, como vem sendo aplicada internacionalmente $e^{16,24-29}$.

\section{Aspectos Gerais da AIS}

Embora não seja uma ideia nova que a saúde pública seja afetada por uma ampla gama de políticas econômicas e sociais ${ }^{30}$, é possível dizer que é nova a percepção atual de que a saúde deve ser considerada explicitamente, ao se avaliarem as políticas públicas. Essa postura é adotada por muitos países desenvolvidos, destacando-se as instituições de saúde como a OMS e o Serviço Nacional de Saúde do Reino Unido (UK-NHS) 1,31-35. Em análise, estão as possíveis consequências à saúde das inúmeras atividades do setor público e privado, sobretudo aqueles envolvidos com: comércio, habitação, transporte, trabalho, energia, educação, entre outros; e suas implicações no cumprimento das metas oficiais de melhoria da saúde da população e de redução das disparidades sociais em saúde.

A AIS pode assumir algumas diferentes definições, no entanto, é comum a ideia de que seja uma ferramenta de tomada de decisão, projetada para levar em conta a grande variedade de potenciais efeitos que uma determinada proposta pode ocasionar sobre a saúde de sua população-alvo, resultando em um conjunto de recomendações baseadas em evidências que auxiliem o processo de tomada de decisão ${ }^{14,17,23,36,37}$. Permite, também, que a saúde seja considerada desde o início do processo de desenvolvimento das políticas ou projetos, ajudando a garantir que os impactos na saúde não sejam negligenciados ${ }^{38-40}$.

Em 2014, foi publicado um guia de referência à AIS pelo Ministério da Saúde ${ }^{41}$, mostrando que a publicação nacional é recente e sua divulgação e aplicação é apoiada pelos órgãos públicos federais. Além dessa publicação, há mais um livro que aborda essa metodologia, intitulado 'O Estudo do Impacto à Saúde Humana na Avaliação de Impacto Ambiental', publicado em 201542. Apesar de o tema de AIS ser incipiente no Brasil, alguns artigos nacionais já apontavam para a introdução da Saúde nos EIARIMAs, mostrando a necessidade de se construir um padrão de análise dos efeitos à saúde aplicável a grandes projetos de desenvolvimento $13,15,18$.

A AIS é regida por alguns princípios que garantem a sua aplicação e, no Brasil, eles podem ser contextualizados aos princípios do SUS, o que permite a sua convergência aos interesses nacionais do setor Saúde (Quadro 1).

Em seu estudo, Silveira et al. ${ }^{15}$ mostram a importância da participação do setor saúde nos processos de licenciamento ambiental. Discutem, ainda, que as avaliações de impactos ambientais em grandes empreendimentos não contemplam a saúde de forma sistemática e harmonizada com os princípios da promoção da saúde e da sustentabilidade socioambiental. Os autores destacam que os impactos na saúde deveriam ser considerados dentro da legislação em que se aplica o EIA-RIMA, ou seja, durante o licenciamento ambiental, o que é muito pouco realizado, também corroborado por Barbosa et al. ${ }^{37}$, Balby et al. ${ }^{17}$ e Drewry e Kwiatkowski ${ }^{14}$. Adicionalmente, apontam que, no Brasil, ainda não há legislação específica que estabeleça participação do setor saúde nos processos de licenciamento ambiental, exceto aquelas vinculadas às áreas endêmicas de malária e em projetos de assentamentos e reforma agrária. Dessa forma, os autores sugerem, assim como os demais trabalhos levantados, a inserção da metodologia da AIS como proposta de introduzir a perspectiva da saúde em políticas públicas, licenciamento ambiental, programas e projetos. 
Quadro 1. Princípios norteadores aplicados à AIS e ao SUS.

\begin{tabular}{|c|c|c|}
\hline Princípio & Aplicação na AIS & Aplicação no SUS \\
\hline Democracia & $\begin{array}{l}\text { A AIS deve envolver e mobilizar o público, informar e } \\
\text { influenciar os tomadores de decisão. Deve ser exposto aqueles } \\
\text { que voluntariamente se sujeitam a riscos e aqueles que estão } \\
\text { involuntariamente expostos aos riscos. }\end{array}$ & $\begin{array}{l}\text { Universalidade de acesso aos serviços de } \\
\text { saúde em todos os níveis de assistência. }\end{array}$ \\
\hline Equidade & $\begin{array}{l}\text { A AIS deve considerar a distribuição dos impactos na } \\
\text { saúde pela população, dando especial atenção aos grupos } \\
\text { vulneráveis, e propor formas de melhorar o desenvolvimento } \\
\text { proposto para os grupos afetados. }\end{array}$ & $\begin{array}{l}\text { Igualdade da assistência à saúde, sem } \\
\text { preconceitos ou privilégios de qualquer } \\
\text { espécie. }\end{array}$ \\
\hline $\begin{array}{l}\text { Desenvolvimento } \\
\text { Sustentável }\end{array}$ & $\begin{array}{l}\text { A AIS deve avaliar os impactos de curto e de longo prazo } \\
\text { de uma proposta, e fornecer essa avaliação aos tomadores } \\
\text { de decisão. Uma boa saúde é a base de resiliência das } \\
\text { comunidades humanas que suportam os processos de } \\
\text { desenvolvimento. }\end{array}$ & $\begin{array}{l}\text { Integração em nível executivo das ações } \\
\text { de saúde, meio ambiente e saneamento } \\
\text { básico; integralidade de assistência. }\end{array}$ \\
\hline Ética & $\begin{array}{l}\text { A AIS deve utilizar as evidências na avaliação de impactos } \\
\text { e na preparação das recomendações; deve ser rigorosa e } \\
\text { transparente e não deve ser usada apenas para apoiar ou } \\
\text { recusar qualquer proposta. }\end{array}$ & $\begin{array}{l}\text { Direito à informação, às pessoas assistidas, } \\
\text { sobre sua saúde; divulgação de informações } \\
\text { quanto ao potencial dos serviços de saúde e } \\
\text { a sua utilização pelo usuário. }\end{array}$ \\
\hline $\begin{array}{l}\text { Abordagem } \\
\text { Global }\end{array}$ & A AIS deve ser guiada pelos indicadores de saúde. & $\begin{array}{l}\text { Utilização da epidemiologia para o } \\
\text { estabelecimento de prioridades, a alocação } \\
\text { de recursos e a orientação programática. }\end{array}$ \\
\hline $\begin{array}{l}\text { Envolvimento das } \\
\text { comunidades }\end{array}$ & Prevê participação da comunidade. & Prevê participação da comunidade. \\
\hline
\end{tabular}

Fonte: elaborado pelos autores com base em Quigley ${ }^{43}$, Ministério da Saúde ${ }^{41}$; Lei 8.080 de $1990^{44}$.

Drewry e Kwiatkowski ${ }^{14}$ reforçam a inserção da AIS nos países da América do Sul e Caribe, mas com a devida capacitação dos profissionais de saúde e da área ambiental, diminuindo, assim, a dependência de especialistas estrangeiros e reforçando ações de coordenação interdisciplinares e redes de trabalho em AIS nacionais e internacionais. A importância da capacitação dos profissionais também é citada na maioria dos artigos levantados e é apontada como uma forma de melhoria do desempenho da gestão socioambiental, pela inclusão da saúde e seus determinantes, resultando em uma melhor utilização dos recursos ${ }^{15,17,36,37}$. Capacitar e ampliar o conhecimento a respeito da metodologia é um ponto importante para garantir que ela não seja realizada de forma superficial. Outro ponto interessante a se levantar é a nomenclatura dessa metodologia no Brasil, uma vez que, nas bases de dados, o descritor é nomeado como 'Avaliação do Impacto na Saúde', entretanto, nas publicações brasileiras é denominado de 'Avaliação de Impacto à Saúde', tanto nos artigos, quanto em trabalhos de doutorado e mestrado, o que mostra a baixa divulgação e padronização dos termos em português, apesar de essa metodologia já ser aplicada há quase duas décadas em países desenvolvidos.

\section{Determinantes de Saúde e o SUS}

Para a adequada aplicação da AIS, deve haver o envolvimento das partes interessadas e a consideração do conceito amplo de saúde, descrito pela OMS como sendo um estado de bem-estar físico, social e psicológico ${ }^{20}$. Esse conceito abrange diversos determinantes de saúde, tais como: condições de habitação, transportes, infraestrutura, nutrição e lazer da população envolvida. Nesse sentido, a saúde é um recurso para a vida, não o objeto de viver. É um conceito positivo, enfatizando recursos sociais e pessoais, bem como capacidades físicas ${ }^{17,40}$.

O investimento em uma abordagem de atenção à saúde consegue oferecer benefícios em, pelo menos, três áreas: aumentar a prosperidade e desenvolvimento econômico, devido ao estado mais saudável e maior disposição dos indivíduos; redução das despesas com problemas de saúde e sociais; 
e estabilidade social, juntamente com o bem-estar da população. Com isso em mente, é capaz de se entender como o ambiente, a economia e a comunidade estão inter-relacionados com a saúde ${ }^{45}$.

Ao se analisar uma população, é necessário considerar os determinantes potenciais em saúde no processo da AIS. Saúde e promoção à saúde são valores sociais amplamente divulgados, mas uma das motivações em realizar a AIS é a compreensão de que as condições econômicas, sociais e ambientais também possuem grande influência na saúde da população 14,15,23,37,46-48. Determinantes em saúde, dessa forma, podem ser qualquer fator pessoal, social, econômico ou ambiental que afete a saúde dos indivíduos de uma população ${ }^{16,40}$. Considerá-los possibilita-se ir ao encontro da definição de saúde, considerada na Lei 8.080 de 19 de setembro de 1990, que regulamenta o SUS, Art. 3º, no qual se descreve que a saúde tem como fatores determinantes e condicionantes, entre outros: a alimentação, a moradia, o saneamento básico, o meio ambiente, o trabalho, a renda, a educação, o transporte, o lazer e o acesso aos bens e serviços essenciais; os níveis de saúde da população expressam a organização social e econômica do País ${ }^{44}$.

Alguns determinantes em saúde podem ser afetados por decisões pessoais, políticas e sociais, formando uma rede inter-relacionada ${ }^{49}$. Nesse âmbito, é fundamental a inserção da AIS em licenciamentos ambientais, projetos e políticas públicas, garantindo a análise dos determinantes de saúde na etapa de planejamento de decisões abrangentes, evitando a sobrecarga ao SUS e facilitando a adoção de medidas mitigadoras no início do planejamento ${ }^{36}$. Além disso, a AIS prevê a potencialização dos efeitos benéficos à saúde de cada proposta, o que favorece a população e o sistema de saúde como um todo $16,17,23$.

\section{Conclusões}

A revisão de literatura é reconhecida por estabelecer relações com produções anteriores, auxilia em apontar novas perspectivas, consolidando uma área de conhecimento específica, e facilita, ao pesquisador, a elaboração de uma síntese do conhecimento existente sobre o assunto. Nessa revisão, constatou-se que há poucas publicações que envolvam a aplicação da AIS na América Latina, o que sugere que a prática e a aplicação da AIS não é muito comum nessa região, ao contrário do que constatado em países desenvolvidos. As publicações também discutem que a análise de impactos à saúde é realizada de forma superficial em licenciamentos ambientais ${ }^{15,41}$ e em políticas públicas nacionais ${ }^{23}$, surgindo a necessidade de formas mais detalhadas e robustas de análises $13-15,18,19,23,37$. A institucionalização surge como uma sugestão para regulamentar as análises em saúde ${ }^{14}$, conforme publicado pelo Ministério da Saúde ${ }^{41}$.

A revisão também apontou que os determinantes em saúde são negligenciados em projetos e políticas ${ }^{36}$, o que também afeta os custos em saúde. Por isso, avaliar quais são e como se interrelacionam os fatores que influenciam a eficiência, a efetividade e a equidade da saúde da população, e seus desdobramentos e impactos no desempenho e responsabilidades do SUS, pode levar a uma melhoria na formulação de políticas e possibilitaria monitorar as desigualdades no acesso e na qualidade dos serviços recebidos pelos diferentes grupos sociais no Brasilio.

A adoção de uma metodologia de impacto à saúde, estruturada e padronizada, como a AIS1, poderia diminuir a geração de efeitos adversos à saúde, decorrentes de projetos e políticas públicas no Brasil|14,15,23, os quais usualmente, desconsideram uma visão de médio e longo prazo. Essa inovação na análise sistêmica de impactos totais poderia poupar muitas vidas e incapacitações, além dos recursos financeiros adjacentes, e, ainda, aumentar a qualidade de vida da população. 


\section{Colaboradores}

As autoras Karina Camasmie Abe e Simone Georges El Khouri Miraglia participaram da elaboração conceitual, delimitação do objeto e do desenho do estudo. Ambas atuaram no levantamento dos dados, discussão, e na confecção do artigo, bem como revisaram e aprovaram a sua versão final.

\section{Agradecimentos}

Esse trabalho recebeu o apoio financeiro da Secretaria de Vigilância em Saúde (SVS) do Ministério da Saúde do Brasil, da Coordenação de Aperfeiçoamento de Pessoal de Nível Superior (CAPES) e do Conselho Nacional de Desenvolvimento Científico e Tecnológico (CNPq).

\section{Referências}

1. WHO. Gothenburg consensus paper: health impact assessment: main concepts and suggested approach [Internet]. [citado 10 Nov 2014]. Disponível em: http://www.apho. org.uk/resource/item.aspx?RID $=44163$.

2. Mindell JS, Boltong A, Forde I. A review of health impact assessment frameworks. Public Health. 2008; 122(11):1177-87. doi: 10.1016/j.puhe.2008.03.014.

3. Scott-Samuel A. Health impact assessment - theory into practice. J Epidemiol Community Health. 1998; 52(11):704-5.

4. Krieger N, Northridge M, Gruskin S, Quinn M, Kriebel D, Davey Smith G, et al. Assessing health impact assessment: multidisciplinary and international perspectives. J Epidemiol Community Health. 2003; 57(9):659-62.

5. Caldwell L. Environmental impact analysis (EIA): origins, evolution, and future directions. Rev Policy Res. 1988; 8(1):75-83. doi: 10.1080/07349165.1988.9725648.

6. Egler PCG. Improving the environmental impact assessment in Brazil. Norfolk, England: Environmental Sciences School, University of East Anglia; 1998.

7. Viegas CV. Atividades de gestão do conhecimento na elaboração do estudo de impacto ambiental [tese]. Florianópolis (SC): Universidade Federal de Santa Catarina; 2009.

8. Ab' Sáber AN, Müller-Plantenberg CM. Previsão de impactos: o estudo de impacto ambiental no leste, oeste e sul. Experiências no Brasil, na Rússia e na Alemanha. São Paulo: Editora USP; 2002.

9. Conselho Nacional do Meio Ambiente (BR). Resolução Conama n001, de 23 de Janeiro de 1986. Dispõe sobre critérios básicos e diretrizes gerais para a avaliação de impacto ambiental. Diário Oficial da União, 17 Fev 1986 [citado 01 Jul 2016]. Disponível em: http://www.mma.gov.br/port/conama/legislacao/CONAMA_RES_CONS_1986_001. pdf.

10. Casa Civil (BR). Lei $n^{\circ} 6.938$, de 31 de Agosto de 1981. Dispõe sobre a Política Nacional do Meio Ambiente, seus fins e mecanismos de formulação e aplicação, e dá outras providências [citado 01 Jul 2016]. Disponível em: http://www.planalto.gov.br/ ccivil_03/Leis/L6938.htm.

11. Conselho Nacional do Meio Ambiente (BR). Resolução Conama n 9 , de 3 de dezembro de 1987. Dispõe sobre a realização de Audiências Públicas no processo de licenciamento ambiental. Diário Oficial da União, 5 Jul 1990 [citado 01 Jul 2016]. Disponível em: http://www.mma.gov.br/port/conama/legiabre.cfm?codlegi=60. 
12. Conselho Nacional do Meio Ambiente (BR). Resolução Conama n 237, de 19 de dezembro de 1997. Dispõe sobre licenciamento ambiental; competência da União, Estados e Municípios; listagem de atividades sujeitas ao licenciamento; Estudos Ambientais, Estudo de impacto Ambiental e Relatório de Impacto Ambiental [Internet]. [citado 01 Jul 2016]. Disponível em: http://www.mma.gov.br/port/conama/res/res97/res23797.html.

13. Barbosa EM, Barata MMdL, Hacon SdS. A saúde no licenciamento ambiental: uma proposta metodológica para a avaliação dos impactos da indústria de petróleo e gás. Cienc Saude Colet. 2012; 17(2):299-310.

14. Drewry J, Kwiatkowski R. The role of health impact assessment in advancing sustainable development in Latin America and the Caribbean. J Environ Health. 2015; 77(8):16-20.

15. Silveira $M$, Neto MDA. Licenciamento ambiental de grandes empreendimentos: conexão possível entre saúde e meio ambiente. Cienc Saude Colet. 2014; 19(9):3829-38. doi: 10.1590/1413-81232014199.20062013.

16. Bhatia R. Health impact assessment: a guide for practice. Oakland, CA: Human Impact Partners; 2011.

17. Balby CN. Avaliação de impactos à saúde: desenvolvimento internacional e perspectivas no Brasil [dissertação]. São Paulo (SP): Faculdade de Saúde Pública; 2012.

18. Silveira M, Padilha JBD, Schneider M, Amaral PST, Carmo TFM, Franco Netto G, et al. Perspective of the health impact assessment in development projects in Brazil: strategic importance for sustainability. Cad Saude Colet. 2012; 20(1):57-63.

19. Rigotto RM. The inclusion of health in environmental impact studies: case report of a coal-fired power plant in Ceará State. Cienc Saude Colet. 2009; 14(6):2049-59.

20. WHO. Ottawa charter for health promotion. First international conference on health promotion. Ottawa; 1986.

21. Conselho Nacional do Meio Ambiente (BR). Resolução Conama n 286/2001, 30 de agosto de 2001. Dispõe sobre o licenciamento ambiental de empreendimentos nas regiões endêmicas de malária. Diário Oficial da União. 17 Dez 2001.

22. Secretaria de Vigilância em Saúde (BR). Portaria n 47, de 29 de dezembro de 2006. Dispõe sobre a Avaliação do Potencial Malarígeno e o Atestado de Condição Sanitária para os projetos de assentamento de reforma agrária e para outros empreendimentos, nas regiões endêmicas de malária. Diário Oficial da União. 04 Jan 2007.

23. Néspoli GRCB. Avaliação de impacto à saúde: aplicação e contribuição para a integração de políticas setoriais no Estado de Mato Grosso [tese]. São Paulo (SP): Faculdade de Saúde Pública; 2010.

24. Andrew L. Use of health impact assessment in the U.S. 27 case studies, 1999-2007. Am J Prev Med. 2008; 34(3):241-56.

25. Harris PJ, Harris-Roxas BF, Harris E, Kemp LA. Health impact assessment and urbanisation. Lessons from the NSW HIA Project. N S W Public Health Bull. 2007; 18(9-10):198-201.

26. Harris $P$, Harris-Roxas $B$, Harris $E$, Kemp L. Health impact assessment: a practical guide. Sydney: Centre for Health Equity Training, Research and Evaluation (CHETRE); 2007.

27. Harris P, Spickett J. Health impact assessment in Australia: a review and directions for progress. Environ Impact Assessment Rev. 2011; 31(4):425-32. doi: 10.1016/j. eiar.2010.03.002.

28. Haigh F, Baum F, Dannenberg AL, Harris MF, Harris-Roxas B, Keleher $H$, et al. The effectiveness of health impact assessment in influencing decision-making in Australia and New Zealand 2005-2009. BMC Public Health. 2013; 13:1188. doi: 10.1186/1471-245813-1188. 
29. Spickett J, Katscherian D, Harris P. The role of health impact assessment in the setting of air quality standards: an Australian perspective. Environ Impact Assessment Rev. 2013; 43:97-103.

30. Krieger N. Epidemiology and social sciences: towards a critical reengagement in the 21st century. Epidemiol Rev. 2000; 22(1):155-63.

31. WHO. Regional Office for Europe. Health impact assessment: main concepts and suggested approach [citado 10 Nov 2014]. Disponível em: http://www.apho.org.uk/ resource/item.aspx?RID $=44163$.

32. WHO. Health impact assessment: a tool to include health on the agenda of other sectors: current experience and emerging issues in the european region. Technical briefing, regional committee for Europe. Copenhagen; 2002.

33. Parry J, Stevens A. Prospective health impact assessment: pitfalls, problems, and possible ways forward. Br Med J. 2001; 323(7322): 1177-82. doi: 10.1136/ bmj.323.7322.1177.

34. Taylor L. Health impact assessment: a review of reviews. London: National Health Service, Health Development Agency; 2002.

35. Scott-Samuel A, Arden K, Birley M. The merseyside guidelines for health impact assessment. 2a ed. International Health Impact Assessment Consortium. Liverpool; 2001.

36. Soares $C B$, Yonekura $T$, Campos $C M$, Figueiro MF. Evidence in public health: steps to make it real. Nurs Clin North Am. 2014; 49(4):533-44. doi: 10.1016/j.cnur.2014.08.008.

37. Barbosa EM. Avaliação de impacto à saúde como instrumento para o licenciamento ambiental na indústria de petróleo [tese]. Rio de Janeiro (RJ): Fundação Oswaldo Cruz; 2010.

38. Taylor L, Quigley R. Health impact assessment: a review of reviews. London: National Health Service. Health Development Agency; 2002.

39. Ison E. Resource for health impact assessment. Vol 1. London, England: NHS Executive London; 2000:198.

40. WHO. Health promotion glossary. Geneva (WHO): World Health Organization; 1998.

41. Ministério da Saúde (BR). Secretaria de Vigilância Ambiental em Saúde. Departamento de Vigilância em Saúde Ambiental e Saúde do Trabalhador. Avaliação de Impacto à Saúde - AIS: metodologia adaptada para aplicação no Brasil. Brasília; 2014. p. 68.

42. Cunha GF. O estudo do impacto a saúde humana na avaliação de impacto ambiental. Jundiaí: Paco Editorial; 2015.

43. Quigley R, Den-Broeder L, Furu P, Bond A, Cave B, Bos R. Health impact assessment international best practice principles. Fargo, USA: International Association for Impact Assessment; 2006 (IAIA, v. 5).

44. Casa Civil (BR). Lei No 8.080, de 19 de setembro de 1990. Dispõe sobre as condições para a promoção, proteção e recuperação da saúde, a organização e o funcionamento dos serviços correspondentes e dá outras providências. Brasília; 1990.

45. Canada. Canadian Handbook of Health Impact Assessment. Canadá: Canadian Minister of Health; 2004. v. 1-4.

46. Terris M. A social policy for health. Am J Public Health Nations Health. 1968; 58(1):5-12.

47. WHO. Closing the Gap in a Generation: Final Report from Commission on the Social Determinants of Health. Geneva: WHO: World Health Organization; 2008.

48. Graham H. Where is the future in public health? Milbank Q. 2010; 88(2):149-68. 
49. Lock K. Health impact assessment. Br Med J. 2000; 320(7246):1395-8.

50. Viacava F, Almeida C, Caetano R, Fausto M, Macinko J, Martins M, et al. Uma metodologia de avaliação do desempenho do sistema de saúde brasileiro. Cienc Saude Colet. 2004; 9(3):711-24.

Abe KC, Miraglia SGEK. Evaluación de Impacto a la Salud (EIS) en Brasil y América Latina: una herramienta esencial para los proyectos, planes y las políticas. Interface (Botucatu). 2018; 22(65):349-58.

Este artículo presenta una revisión de la literatura y discute la aplicación de la metodología de Evaluación del Impacto a la Salud (EIS), divulgada por la Organización Mundial de la Salud (OMS), para Brasil y América Latina. Ese estudio mostró que la práctica y la aplicación de EIS no es común en esas regiones y, además, el análisis de impactos a la salud se realiza de forma superficial en licencias ambientales y en políticas públicas, lo que señala la necesidad de la utilización de metodologías específicas, capacitación de profesionales e iniciativa gubernamental. La adopción de EIS en Brasil y en América Latina evitaría la generación de efectos adversos a la salud, potencializando los aspectos positivos, mitigando los efectos negativos de proyectos y políticas públicas, garantizando que no se descuide la salud de la población.

Palabras clave: Evaluación del impacto en la salud. Salud pública. Sistema Brasileño de Salud. Gestión en salud. Brasil. 